\title{
RECENT WORK ON FUNDAMENTAL ASTROMETRY IN THE U.S.S.R.
}

\author{
M.S. ZVEREV
}

Absolute determinations of star positions using transit and vertical circles according to the methods developed at Pulkovo are being made at Pulkovo, at Nikolaiev, at the Golossejevo Observatory of the Ukraine Academy of Sciences, and at Santiago de Chile. At this latter observatory Soviet and Chilean astronomers have completed a series of observations of both bright and faint fundamental stars with a photographic vertical circle, and these observations are now being reduced at Pulkovo. Absolute determinations of right ascension will be made at the Cerro Calan Observatory using a large transit instrument recently constructed at Pulkovo. Further details of some of the Southern observations are given in the following paper to be presented by Dr. Anguita.

Independent determinations of right ascension have been made since the time of the International Geophysical Year by the Time Service of the U.S.S.R. using photoelectrically recording instruments and the methods proposed by Professor N. N. Pavlov (1958) and Professor A.A. Nemiro (1957). In these programmes a special effort is made to obtain observations of the zenith stars right through the night in order to derive an independent system of $\Delta \alpha_{\alpha}$.

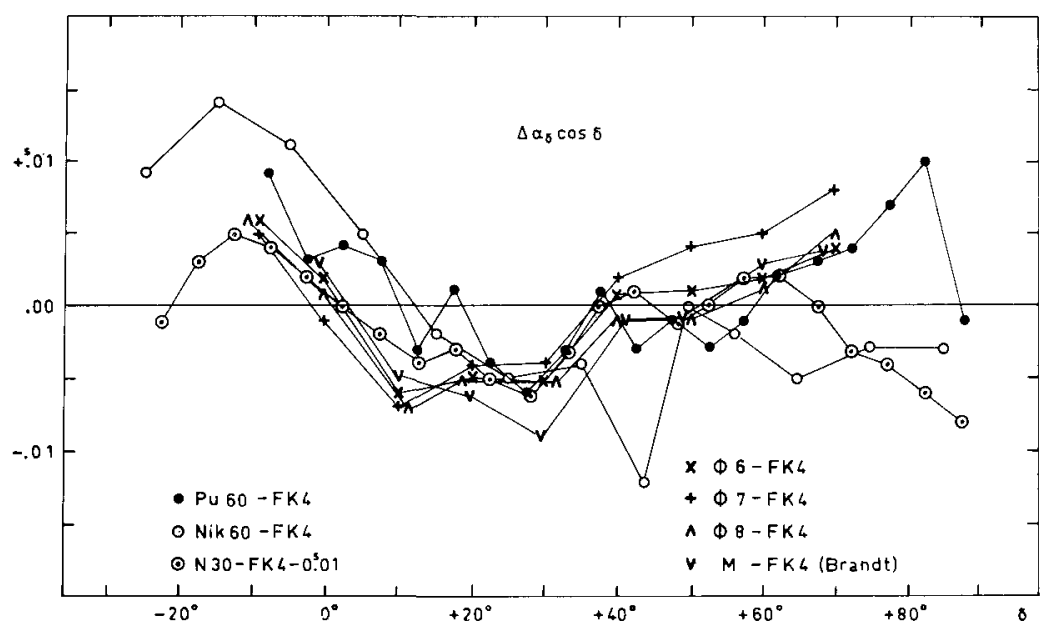

FIG. 1.

Perek (ed.), Highlights of Astronomy, 286-291. (C) I.U.A. 
The azimuth is determined both by combining observations of zenith stars at upper and lower culminations and by combining observations of zenith and equatorial stars. (Pavlov's method is only suitable for latitudes higher than $50^{\circ}$.) The catalogues resulting from these observations of the Time Services seem to be very accurate both as regards systematic and accidental errors (Pulkovo, 1966; Brandt, 1963).

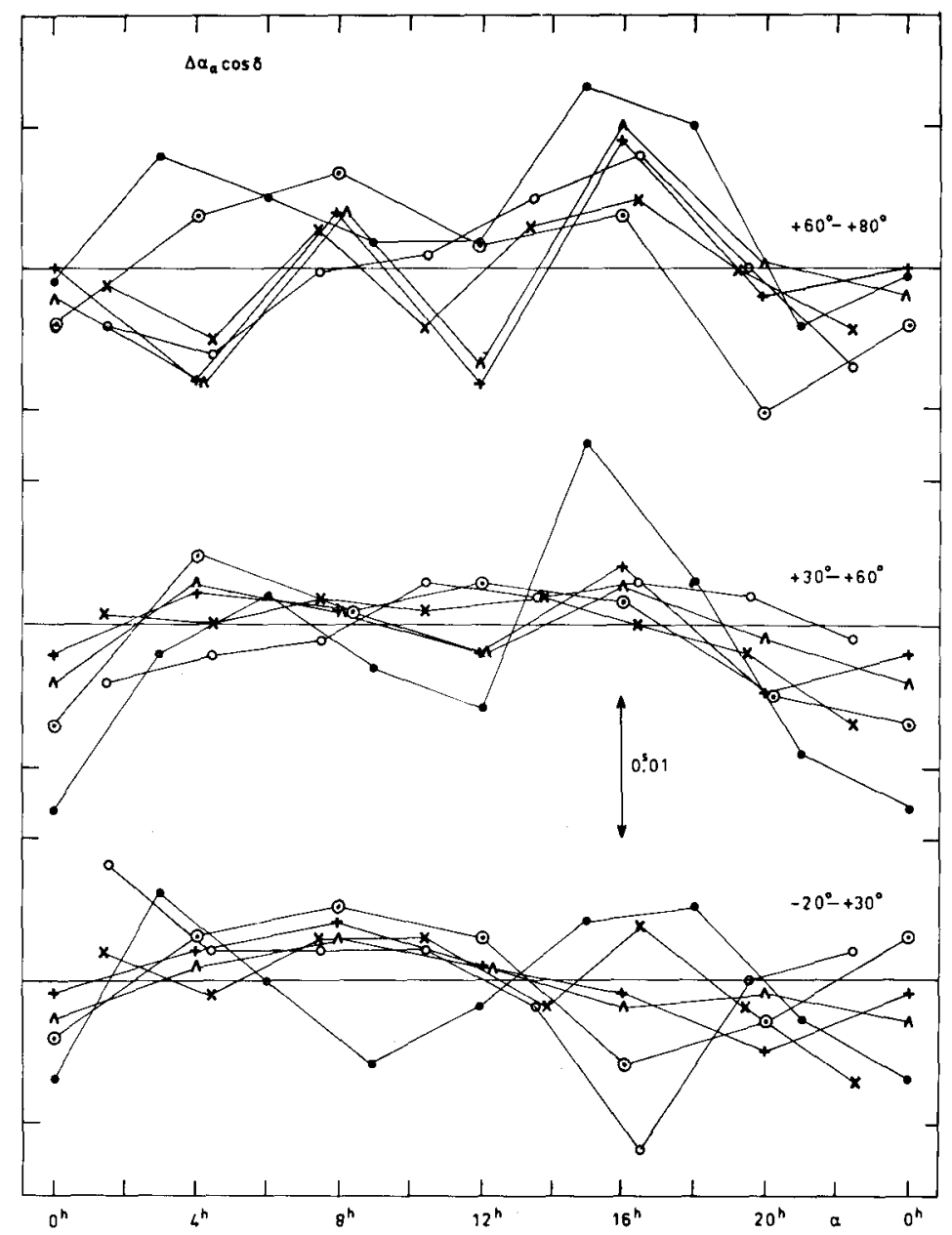

FIG. 2.

Systematic differences at 1960.0 between some of these recent absolute catalogues and FK4 are shown in Figures 1-4, in which the data for Pu 60 must be regarded as preliminary. It will be seen that the new catalogues are in fairly good agreement with one another and that they agree rather better with N30 than with FK4, a fact which 


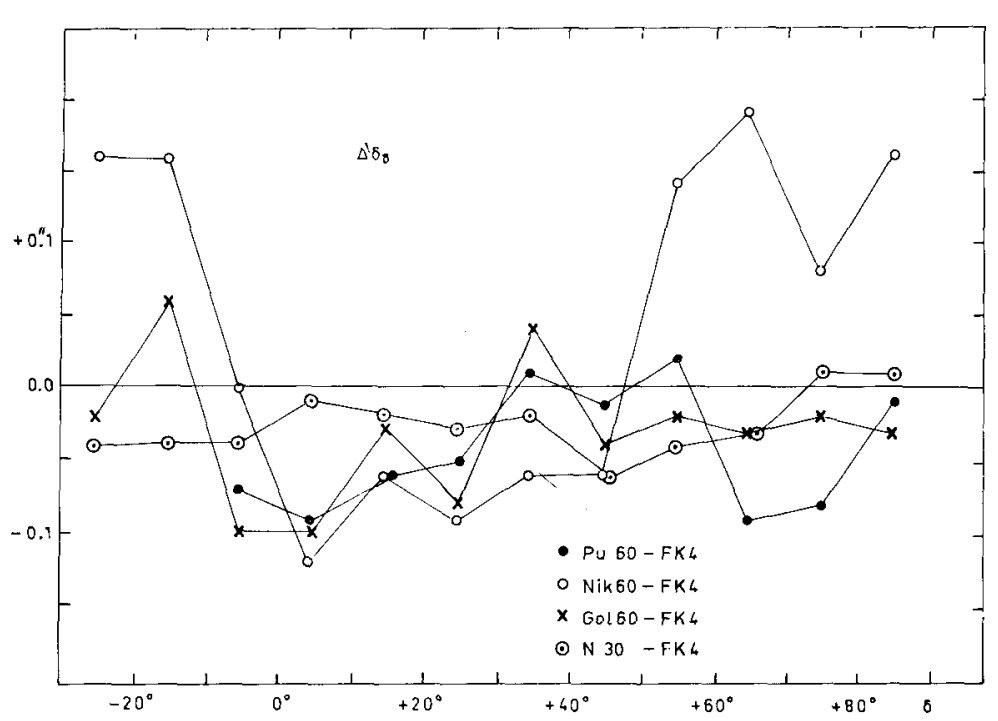

FIG. 3 .

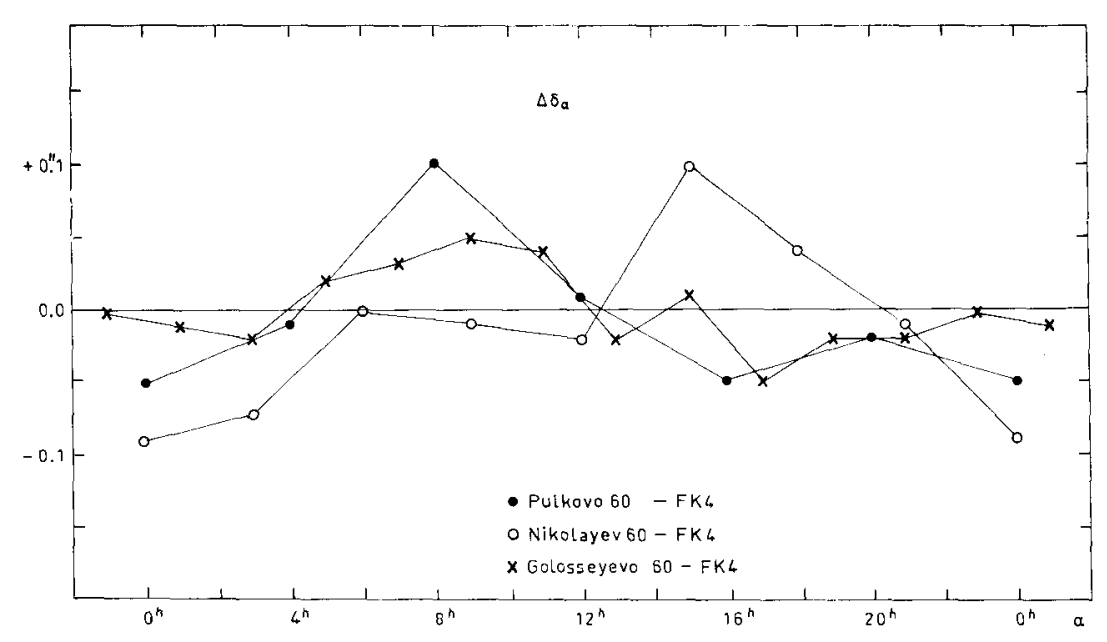

FIG. 4.

seems to justify the methods used by Dr. H. R. Morgan for the determination of proper motions during the compilation of $\mathrm{N} 30$.

As may be seen from Professor Nemiro's report to Commission 8, the Russian observatories are taking their full share in all the international programmes. Observations are also being made of special groups of stars such as those in the areas surrounding the galaxies selected by Professor Deutsch (Bugoslavskaja et al., 1955).

Stars in the North polar area are receiving special attention since they are relatively 
few in number and the systematic errors in their observed positions often differ considerably from those for other zones. About ten catalogues of the right ascensions of North circumpolar stars have recently become available, some of them as a result of the reductions of observations made many years ago, for instance by W. Fabritius at Kiev about 1880. Most of these catalogues can be regarded as absolute so that they should greatly strengthen the right ascension system in the Northern sky.

Observations and investigations in connection with the Catalogue of Faint Stars (KSZ) have been continued along the lines reported by myself to Commission 8 at

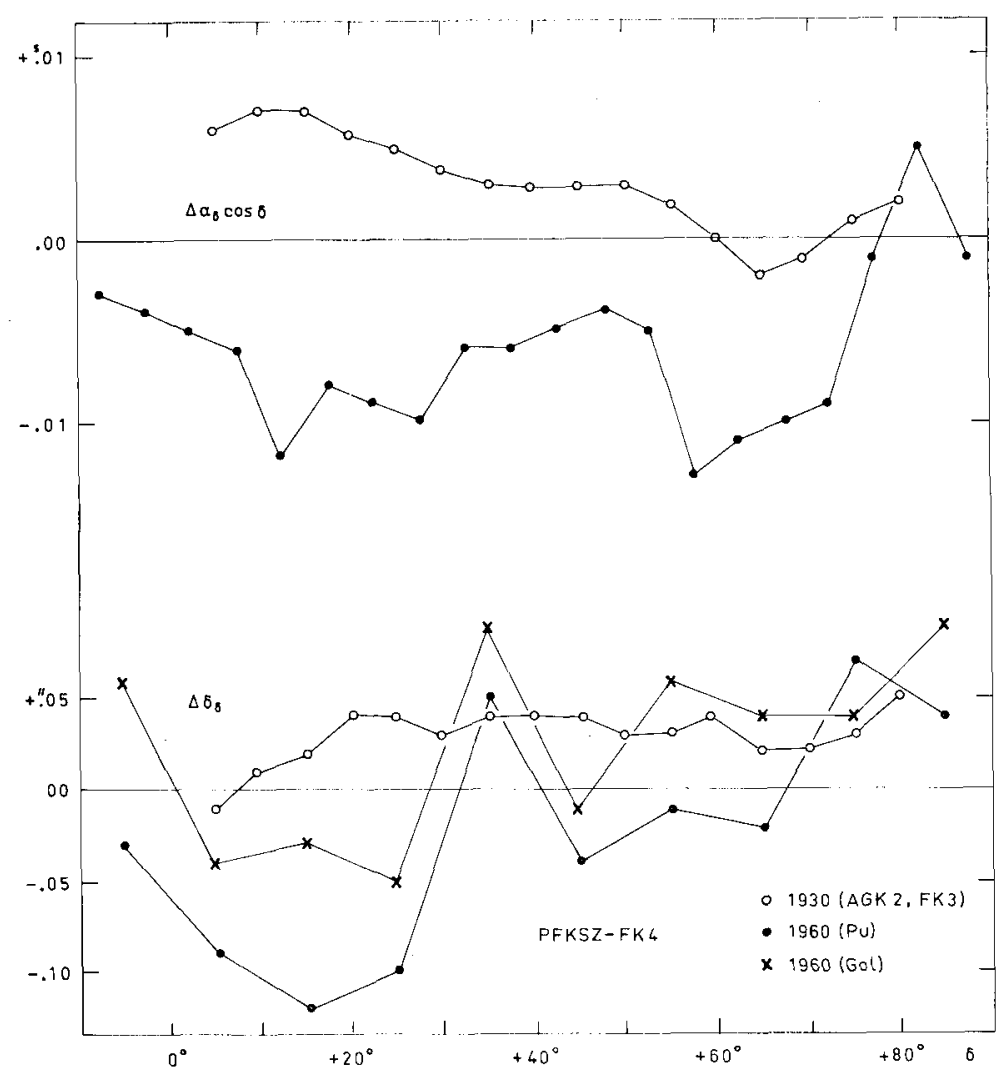

FIG. 5 .

Hamburg (IAU, 1966) and by Dr. V.I. Orelskaja and Professor A. N. Deutsch here in Prague. Later today Dr. N.V. Fatčihin will be telling us about the first Pulkovo results from the programme for obtaining absolute proper motions from observations of galaxies.

Bright stars can be related to the galaxies or to minor planet orbits only through the intermediary of faint stars. The determination of the relationship between the 


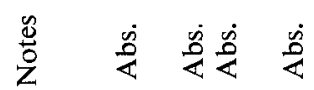

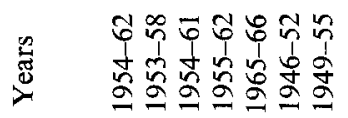

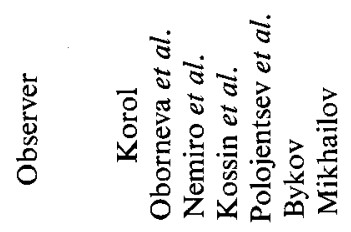

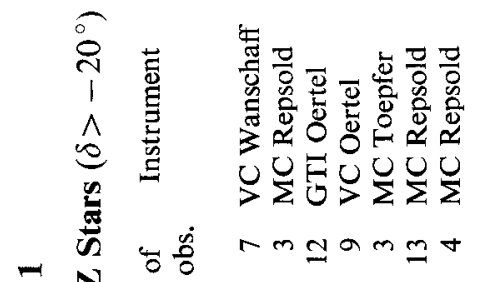

है

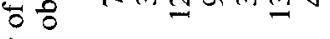

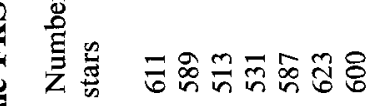

$\pm z$ in

ஜ융요요

$+++++1+$

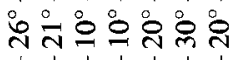

i)

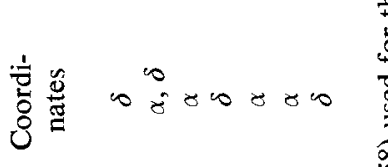

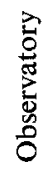

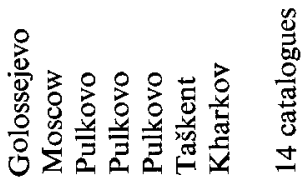

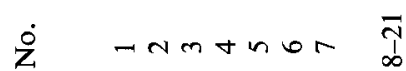

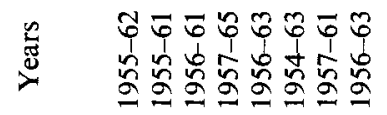

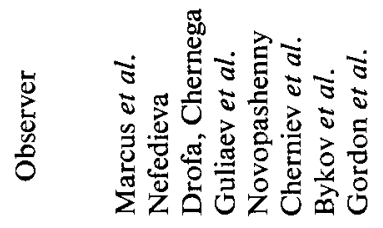

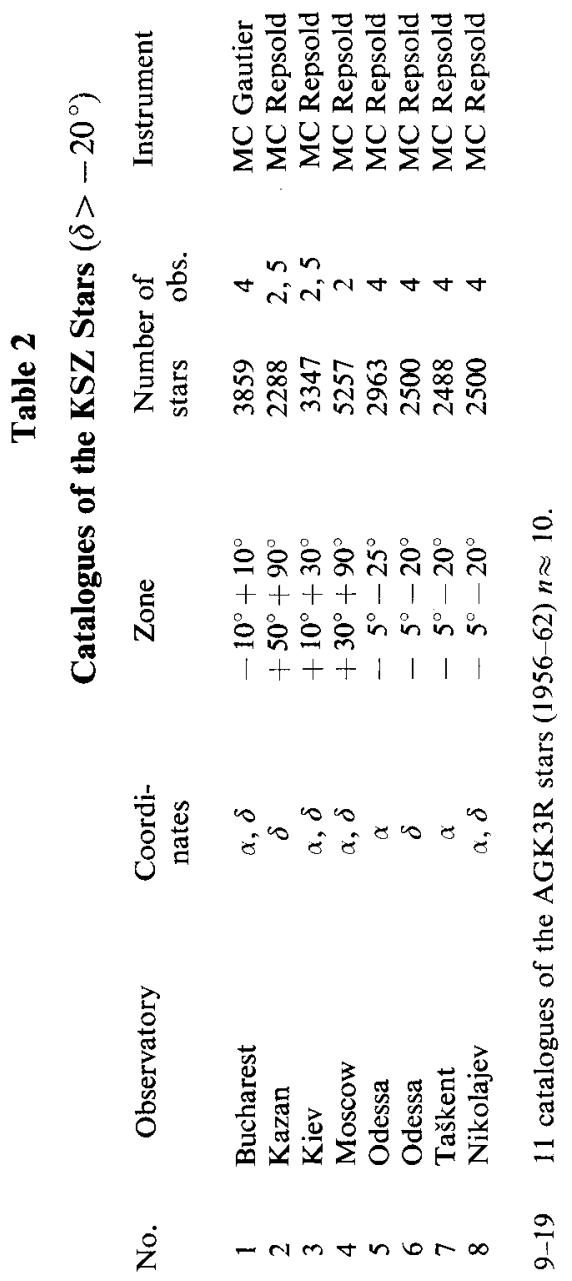


position and proper motion systems of the bright and faint stars is thus one of the most important problems of modern astrometry. As FKSZ and FK4 have no stars in common, their systems must be compared either by direct observation or by using an intermediary catalogue.

We have tried both these methods for a preliminary comparison of the PFKSZ and FK4 systems, but, as may be seen from Figure 5, we did not obtain good results. Figure 5 shows the $\Delta \alpha_{\delta}$ and $\Delta \delta_{\delta}$ systematic differences between PFKSZ and FK4, as determined from new meridian observations made at Pulkovo and Golossejevo, and as determined by using AGK2 (epoch 1930.0) and applying the differences FK3FK4. Part of the lack of agreement between the results obtained from the two methods may possibly be attributed to the assumed proper motions, but not all.

The improvement of the Preliminary Fundamental Catalogue of Faint Stars (PFKSZ), which was compiled about 10 years ago (Pulkovo, 1958), is an important and an urgent task. Tables 1 and 2 indicate what catalogues are available for the revision of the Northern section of the FKSZ and the KSZ. The first includes seven new catalogues, of which four are fundamental, and the second - eight. As the KSZ catalogues include FKSZ stars, there are available for the recompilation of the Northern part of the FKSZ 40 catalogues from 19 observatories with about 90 observations of each star in each coordinate. For the Southern part of the FKSZ, however, the only observations available are those from Cape, La Plata, and Santiago.

The Southern KSZ and FKSZ stars are included in the SRS programme, but observations made for this latter programme will not be sufficient for the compilation of a definitive FKSZ catalogue. I would strongly urge all Southern meridian observers to include the FKSZ stars in all their programmes, particularly in programmes of absolute observations.

\section{References}

Brandt, W.E. (1963) Rotation of the Earth, Kiev, p. 64.

Bugoslavskaja, E.J., Karimova, D. K., Podobed, V.V., Jakhontov, K. N. (1955)

Astrometric Conference of the U.S.S.R., Leningrad, p. 42.

IAU (1966) Trans. IAU, XII-B, p. 124-125.

Nemiro, A.A. (1957) Pulkovo Bull., 157.

Pavlov, N.N. (1958) Pulkovo Bull., 161.

Pulkovo (1958) Publ., 72.

Pulkovo (1966) Publ., 75. 